\title{
Citation:
}

Khatami H.R. and O'Kelly B.C., 2013. Improving mechanical properties of sand using biopolymers. ASCE Journal of Geotechnical and Geoenvironmental Engineering, Vol. 139. doi: 10.1061/(ASCE)GT.1943-5606.0000861

http://ascelibrary.org/doi/abs/10.1061/\%28ASCE\%29GT.1943-5606.0000861

\section{Improving mechanical properties of sand using biopolymers}

Hamid Reza Khatami

B.Sc., M.Sc.

PhD Candidate, Department of Civil, Structural and Environmental Engineering, Trinity College Dublin, Dublin 2, Ireland.

e-mail: khatamih@tcd.ie

Tel. +35318963676

Brendan C. O'Kelly

BE, MEngSc, PhD, FTCD, CEng, CEnv, MICE, FIEI

Associate Professor, Department of Civil, Structural and Environmental Engineering, Museum Building, Trinity College Dublin, Dublin 2, Ireland.

e-mail: bokelly@tcd.ie

Tel. +35318962387

Fax. +35316773072

Number of Tables: 1

Number of Figures: 5

Submitted: 18th February 2012

Accepted: 31st October 2012

Published: 3rd November 2012 


\begin{abstract}
Natural polymers (biopolymers) are discussed as environmentally friendly and sustainable grouting chemicals. This paper presents guidelines for selecting potentially useful biopolymers for strengthening cohesionless soil. Agar and six modified starches were identified for further study over a range of concentrations (1-4\% agar and $0.5-1 \%$ starch). Experimental results demonstrate the compatibility of agar and starch. Depending on biopolymer concentration, the unconfined compressive strength of sand treated with agar and starch biopolymers ranged from 158 to $487 \mathrm{kPa}$. Triaxial compression tests over a range of confining pressures also indicated that the biopolymers effectively increased the cohesion intercept and stiffness of the treated sand.
\end{abstract}

KEYWORDS: biopolymer, compressive strength, ground improvement, sand, shear strength, stiffness.

\title{
1. Introduction
}

The deliberate process of incorporating a dispersed phase capable of binding particles and filling the pore voids in the soil matrix can bring about an overall improvement in mechanical properties and (or) a reduction in hydraulic conductivity of the treated ground. Over the past decades, numerous types of chemicals (e.g., sodium silicate, epoxy, polyurethane, and acrylamides) have been considered for geotechnical application. Chemical grouts are generally toxic and hazardous, with a few notable exceptions, e.g. sodium silicate. This necessitates pursuing comprehensive research in order to find alternative eco-friendly materials for ground treatment. It appears that several natural polymers (biopolymers) can be used as substitutes for conventional components of grouting materials, especially for temporary works and relatively dry conditions. The widespread application of biopolymers in this manner would provide a sustainable industry because they are mainly obtained from the non-food parts of plants being cultivated year on year. Previous research has mainly focused on the effect of biopolymers on soil permeability. Martin et al. (1996) and Karimi (1998) investigated the effects of biopolymers, specifically xanthan gum and sodium alginate, on mainly permeability but also shear strength of a silty soil, reporting a reduction of two orders of magnitude in permeability and a 30\% increase in shear strength. Khachatoorian et al. (2003) showed that some biopolymers, when flooded into the matrix of a petroleum reservoir, decrease the permeability relative to fluids present in the reservoir. Also, Bouazza et al. (2009) applied xanthan gum, guar gum and sodium alginate in concentrations up to $2 \%$ by weight to a silty sand and found that xanthan gum provided better performance in lowering permeability. The application of agar in concentrations of typically less than $1 \%$ to stabilize soil temporarily by coating the soil grains with a biopolymer film has been described by Schneider et al. (1989), Frost (1989) and Sutterer et al. (1996) in the development of undisturbed sampling procedures. The present research investigates the potential of two biopolymers, namely agar and starch, to enhance the strength of sand.

\section{Biopolymer selection}

The biopolymer selection was a two-stage process that considered surface properties and mechanical properties, which are responsible for providing adhesion and cohesion 
respectively. Cohesion refers to the intermolecular attraction by which the elements of a body are held together. In a solid, to the extent that resistance to an outward force or a tension operates to prevent separation of the atoms of a solid it is commonly known as cohesion. Adhesion, on the other hand, is defined as the physico-chemical attraction forces between dissimilar surfaces that make them stick together. Depending on their structure, biopolymers possess various chemical functional groups such as hydroxyl, ester or amines. Their longchain structure also provides frequent sites for the characteristic chemical reaction of a given functional group to occur. Chemical bonding corresponds to the adhesive forces whose function is to hold the soil particle and gel together at their surfaces. At microscopic scale, the effectiveness of the bonding mainly depends on the type of forces present at the interface of the soil particle and the biopolymer gel. The forces operating at such phase-interface include: ionic/electrostatic or covalent bonds (chemisorption); hydrogen bonding (strong polar attraction); van der Waals forces (physical absorption). Short range ionic/electrostatic and covalent bonds have the highest bond energy in terms of $\mathrm{KJ} / \mathrm{mol}$ and therefore give the strongest bond. van der Waals forces, which are the interaction between dipoles within the bulk material, develop the weakest bonds over a long range. In selecting a biopolymer, a cationic biopolymer together with the soil particles would provide strong electrostatic bonding throughout the treated ground because most natural particles in soil show a slight negative surface charge. Alternatively, a chemical capable of forming hydrogen bonding, which is still quite a strong chemical attraction force, with the soil particles would ideally be used in the case of non-ionic biopolymers.

In terms of mechanical properties, polymers vary extensively in mechanical performance depending on their degree of crystallinity, molecular weight and degree of cross-linking (Feldman, 1989). High strength and rigidity are obtained with high degree of crystallinity and/or cross-linking. A biopolymer of adequately regular chemical structure is believed to be capable of undergoing crystallization to some extent. Also, the higher its molecular weight, the greater the chance for ordering and crystallization of its macromolecule chain. The selection of a biopolymer of appropriate molecular weight, and also of a viscosity feasible for injection, is usually a matter of trade-off because the apparent viscosity in water usually increases as the molecular weight increases. The flexibility of the polymeric chain is also believed to be particularly important for permeation of the grout. Once placed within the soil matrix, the biopolymer is desired to undergo some form of cross-linking in order to enhance strength and reduce its mobility in the ground. Cross-linking connects polymeric chains through chemical reactions (initiated by temperature rise, change in pressure or $\mathrm{pH}$ ) and can form a comprehensive lattice in the soil matrix. This rigidifies the whole polymeric structure, thereby enhancing mechanical strength. Flexibility can be regarded as an initial property of a polymeric chain to facilitate the penetration of a polymeric grout into the soil. On the other hand, cross-linking is desired to take place after the grout has permeated the soil void spaces or while it is curing.

Taking the preceding factors into account, the authors decided to focus their efforts on two different types of biopolymers, namely agar and modified starch. Agar is understood to provide the highest mechanical strength among biopolymers and modified starch is positively charged. Hence, together they have the potential to generate significant cohesion and adhesion between the negatively charged sand particles.

Agar is contained in the cell walls of several species of red algae including Gelidium, Pterocladia and Gracilaria. Natural agar consists of a mixture of predominantly agarose but also agaropectin, with the former showing good gelling properties. Agarose starts to dissolve 
at about $85^{\circ} \mathrm{C}$, the exact dissolution temperature depending on the agar source. After dissolution, the viscosity of the agar solution increases constantly with reducing temperature until finally solidifying between $34^{\circ} \mathrm{C}$ and $40^{\circ} \mathrm{C}$. From the range of polysaccharides, agarose is the only one that can form a three-dimensional gel network in the soil matrix, which would produce a dense film, coating the sand grains, after curing. Due to its complex structure, very few bacteria can digest agar gel and hence this attribute minimizes its biodegradation. Agar also shows sensitivity to polymeric additives and unless a suitable additive is used it can significantly reduce strength and chemical resistance. From the authors' perspective, it appears that positively charged starch, which can be manufactured by physical, chemical or even enzymatic processes, is compatible with agar. In contrast, natural starch is either nonionic or slightly anionic, depending on the material source. Starch is a white, tasteless, odorless powder that does not dissolve in cold water or ethanol. It consists of linear and helical amylose along with branched amylopectin which consists of glucose monomers.

\section{Materials and methods}

Fontainebleau sand with a particle size range of 0.06 to $0.4 \mathrm{~mm}$ was used in all experiments. Approximately $90 \%$ of the sand grains by dry mass were in the size range of 0.15 to $0.3 \mathrm{~mm}$. It is a white siliceous sand $\left(\mathrm{SiO}_{2}>99.8 \%\right)$ with sub-angular grains whose relatively high specific surface area and clean surfaces are ideal for the adsorption of biopolymers. The sand has a specific gravity of 2.66, minimum density of $1.39 \mathrm{Mg} / \mathrm{m}^{3}$ and maximum density of 1.75 $\mathrm{Mg} / \mathrm{m}^{3}$, with $\mathrm{D}_{10}=0.15$ and $\mathrm{Cu}=1.6$ where $\mathrm{D}_{10}$ is the equivalent spherical grain size corresponding to $10 \%$ finer by weight, $\mathrm{D}_{60}$ corresponds to $60 \%$ finer by weight, and $\mathrm{Cu}$ is $\mathrm{D}_{60} / \mathrm{D}_{10}$.

The biopolymers used in the present study were microbiological-grade agar from VWR International and six commercial modified starches (Staramic 105 and 747 along with Starpol 136, 469, 600 and 700) from Tate and Lyle. These starches were supplied as pre-gelatinized powder, showing at least $20 \%$ solubility in cold water.

Complete dissolution was achieved by placing containers with the biopolymer mixtures in an oven, set at a temperature of $95^{\circ} \mathrm{C}$ for a period of $2 \mathrm{~h}$. The containers were sealed to prevent loss of water which would otherwise have changed the final concentration of the solution.

The dry sand was air-pluviated into specimen molds, 38- $\mathrm{mm}$ in diameter by 76- $\mathrm{mm}$ long, with the free-fall height of the sand grains adjusted to produce dry densities of 1.50 to 1.54 $\mathrm{Mg} / \mathrm{m}^{3}$ (i.e., relative densities of $36-47 \%$ ). The biopolymer solution was poured into the molds, allowed to permeate through the sand and fill the voids. The specimens were cured at laboratory temperature of $21^{\circ} \mathrm{C}$ over a three-day period followed by oven- drying at $35^{\circ} \mathrm{C}$ over a four-day period, after which strength tests were performed in triaxial compression. The mass ratio of biopolymer to dry sand for all of the test-specimens ranged from $0.3 \%$ to $1.2 \%$. The research was carried out in two stages. Firstly, trials were performed on a range of biopolymer combinations and concentrations to identify which of the treatments were more effective in improving the strength properties of the sand. Secondly, unconfined compression and unconsolidated-undrained triaxial compression tests were performed at a strain rate of $0.01 \% / \mathrm{min}$, with confining pressures of 100,200 and $400 \mathrm{kPa}$, in order to identify a suitable modified starch for use in combination with agar. The test results also provided necessary data for determining the Mohr-Coulomb parameter values. 


\section{Experimental results and discussion}

A series of triaxial compression tests were performed on the dry sand and sand that had been treated with solutions containing $1 \%, 2 \%$ and $4 \%$ of agar by weight. The data indicated that higher agar concentrations produced higher compressive strength and stiffness for the treated sand (Figure 1). The maximum deviatoric stress, mobilized for a given confining pressure, also increased at higher agar concentrations (Figure 2).

Since agar gel and sand particles are both slightly negative in charge, it would appear a valuable practice of adding a positively charged intermediate agent, such as modified starch, to further enhance the biopolymer treatment. Figure 3 shows deviatoric stress-strain responses of the sand treated with mixtures of $1.0 \%$ agar and $0.5 \%$ of the different starches.

From Figure 3, the following are inferred for the starches considered in this study:

(i) In general, most starches increased compressive strength and stiffness remarkably;

(ii) Starpol 136 produced the greatest increase in strength followed by Starpol 469, although the latter demonstrated significantly higher ductility;

(iii) Staramic 105 and Starpol 700 were found to marginally reduce compressive strength and also the strain at failure compared to agar alone.

Figure 4 shows the deviatoric stress-strain responses of the sand treated with $1.0 \%$ agar and either $1.0 \%$ Starpol 136 or $0.5 \%$ Starpol 600 , which were two of the better performing starches, mobilizing greater deviatoric stress compared with sand treated with agar alone (Figure 1). Starpol 136 produced a higher deviatoric stress for a given confining pressure, although the concentration of the former was twice that of the latter.

The test-specimens failed in compression either by bursting and disintegration into sand clusters, by forming a rough shear plane or an intermediate state for sand treated with $1.0 \%$, $2.0 \%$ and $4.0 \%$ agar solution, respectively (Figure 5(a-c)). Also, the specimen treated with $1.0 \%$ agar and $0.5 \%$ Starpol 600 sheared along a distinct failure plane at a slightly lower axial strain (Figure 5(d)). It is postulated that since agar gel by itself behaves plastically, increasing its concentration introduces some degree of ductility to the treated soil matrix. Addition of $0.5 \%$ starch had the effect of increasing brittleness, and it is consistent with the nature of starch solution, which sets into a brittle solid at concentrations greater than $15 \%$.

Two mathematical frameworks were used to estimate the Mohr-Coulomb parameters of cohesion intercept, $c$, and friction angle, $\varphi$, namely non-linear optimization and linear leastsquares analyses of the maximum deviatoric stress (Eqs. (1) and (2) respectively):

$$
\begin{aligned}
& \frac{\sigma_{1 \mathrm{f}}}{\sigma_{3 \mathrm{f}}}=\frac{2 \cdot c \cdot \cos \varphi}{\sigma_{3 f}(1+\sin \varphi)}+\frac{1+\sin \varphi}{1-\sin \varphi} \\
& c=b \cdot \frac{1-\sin \varphi}{2 \cos \varphi} \\
& \varphi=\arcsin \left(\frac{m-1}{m+1}\right)
\end{aligned}
$$

where $\sigma_{l f}$ and $\sigma_{3 f}$ denote the major and minor principal stresses at failure, respectively. 
For the non-linear optimization, the Solver tool of Microsoft Excel was used to find the responses for $c$ and $\varphi$ of this mathematically over-determined system (Table 1).

In the linear least-squares analysis, data of $\left(\sigma_{3 f}, \sigma_{l f}\right)$ were plotted in a $\sigma_{3}-\sigma_{1}$ plane and fitted by the best line of

$$
\sigma_{1}=m \cdot \sigma_{3}+b
$$

The relationships between $c$ and $\varphi$ with slope, $m$, and intercept, $b$, of this best-fit line are given by Eqs. (2a) and (2b). Table 1 indicates that the cohesion intercept was directly proportional to the concentration of agar. Furthermore, the addition of starch at the same agar concentration substantially increased the cohesion intercept; for instance, by threefold for $0.5 \%$ Starpol 600 and fourfold for $1 \%$ Starpol 136. However, the biopolymer treatment was found to produce a step reduction in $\varphi$ from $32-33^{\circ}$ for the untreated sand to $25-26^{\circ}$ for sand treated with $1-4 \%$ agar solution. The addition of starch produced a further step reduction in $\varphi$ to $17.5^{\circ}$. It is postulated that the coating effect of the biopolymer on the grain surfaces smoothened the micro-scale roughness, thereby reducing the asperity interlocking of the sand grains.

\section{Summary}

Biopolymers can effectively improve the strength characteristics of sand without causing environmental toxicity. The improvement in performance of sand treated with agar and modified starch was found to be directly dependant on the concentration of agar as the main component and starch as the additive. The addition of Starpol 600 and 136 at the same agar concentration was observed to significantly increase the value of cohesion intercept and to also enhance stiffness.

In conclusion, biopolymer treatment shows promise as a tool to modify and engineer soil behavior to have specific strength and deformation characteristics in terms of level of stiffness or ductility. The sustainability and eco-friendliness of biopolymers also add to their attractiveness for use in engineering applications.

\section{Acknowledgments}

The first author would like to acknowledge the Irish Research Council (IRC) for financial support under the Embark Postgraduate Research Scholarship Scheme 2010 (RS 2010/2571). Special thanks are also due to Mr. Marin Carney and Mr. Eoin Dunne for assistance in performing the laboratory tests.

\section{References}

Bouazza, A.,Gates, W. P., \& Ranjith, P. G. (2009). "Hydraulic conductivity of biopolymer treated silty sand." Géotechnique, 59(1), 71-72.

Feldman, D. (1989). "Polymeric Building Materials.” Elsevier Science Publishers. 
Frost, J. D. (1989). "Studies on the monotonic and cyclic behavior of sands." Technical Report, Purdue University, West Lafayette, IN

Khachatoorian, R., Petrisor, I. G., Kwan, C. C. \& Yen, T. F. (2003). "Biopolymer plugging effect: laboratory-pressurized pumping flow studies.” J. Pet. Sci. Engng, 38(1-2), 13-21.

Martin, G. R., Yen, T. F., and Karimi, S. (1996). "Application of biopolymer technology in silty soil matrices to form impervious barriers", Proceeding of 7th Australia-New Zealand Geomechanics Conference, Adelaide, Australia.

Schneider, H. R., Chameau, J.-L., \& Leonards, G. A. (1989). "Chemical impregnation of cohesionless soils.” ASTM Geotechnical Testing Journal, 12(3), 204-210.

Sutterer, K. G., Frost, D. J., \& Chameau, J.-L. A. (1996). "Polymer impregnation to assist undisturbed sampling of cohesionless soils." J. Geotech. Engrg, 122(3) 209-215. 
Table 1. Mohr-Coulomb parameters for sand treated with biopolymers

\begin{tabular}{|l|c|c|c|c|}
\hline \multirow{2}{*}{ Conditions } & \multicolumn{2}{|c|}{ Non-linear optimization } & \multicolumn{2}{c|}{ Linear least squares } \\
\cline { 2 - 5 } & $c(\mathrm{kPa})$ & & $c(\mathrm{kPa})$ & \\
\hline Untreated sand & 0 & 32.3 & 0 & 33.1 \\
\hline $1 \%$ agar & 62 & 24.7 & 49 & 25.4 \\
\hline $2 \%$ agar & 111 & 25.6 & 105 & 26.4 \\
\hline $4 \%$ agar & 190 & 26.3 & 222 & 23.6 \\
\hline $1 \%$ agar and $0.5 \%$ Starpol 600 & 187 & 17.5 & 187 & 17.5 \\
\hline $1 \%$ agar and $1 \%$ Starpol 136 & 240 & 17.6 & 245 & 17.4 \\
\hline
\end{tabular}




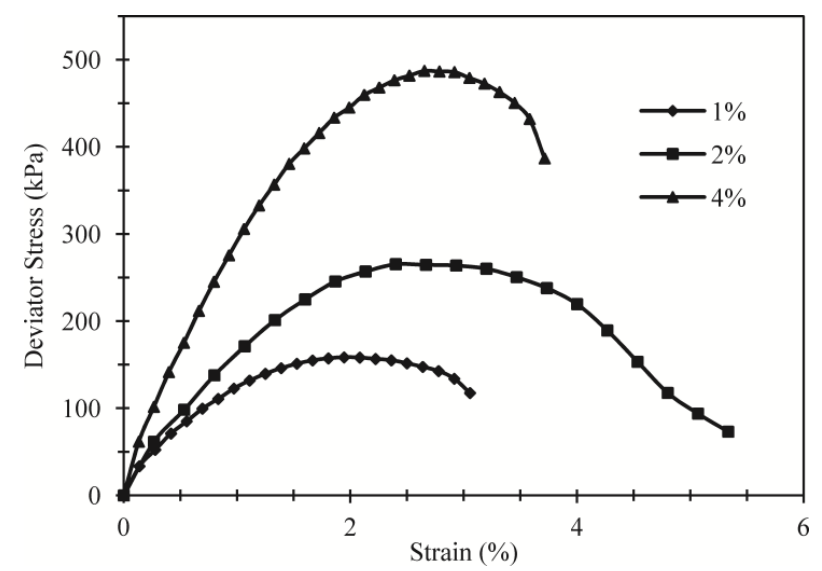

Fig. 1. Unconfined compression tests of sand treated with agar

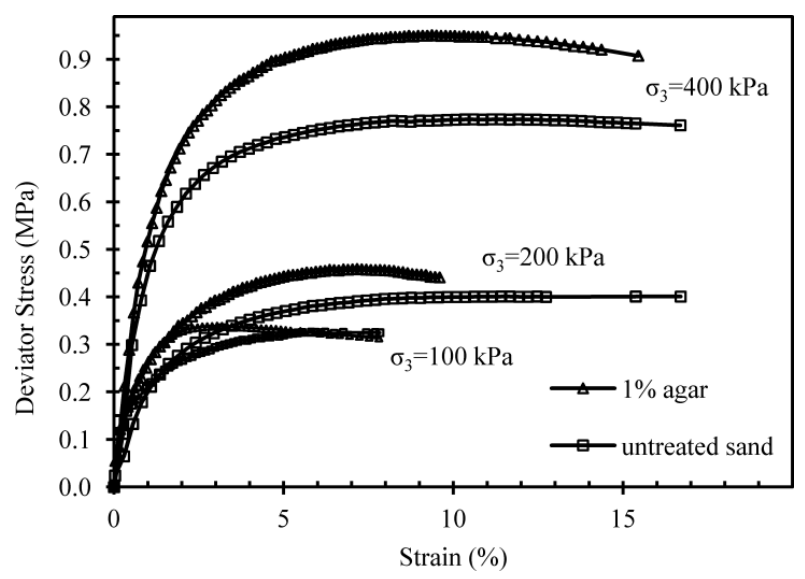

Fig 2 (a). Triaxial compression tests on untreated sand and sand treated with $1 \%$ agar grout

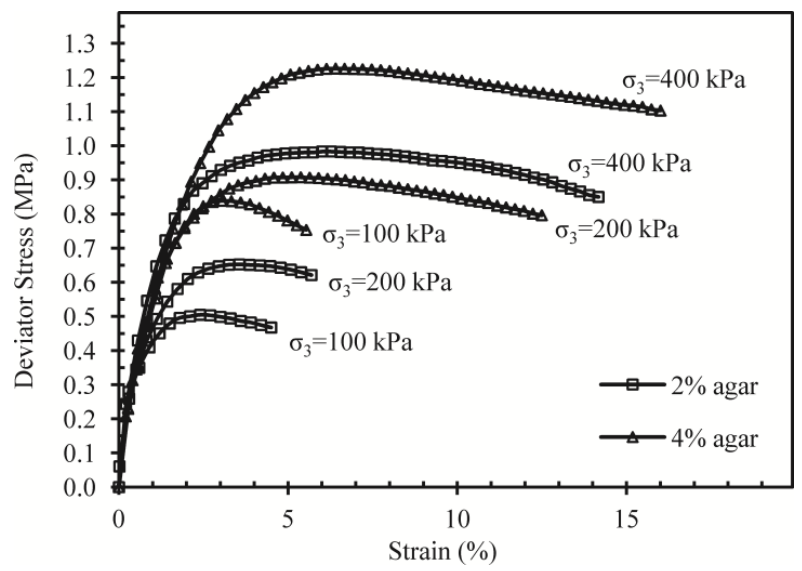

Fig 2 (b). Triaxial compression tests on untreated sand and sand treated with $2 \%$ and $4 \%$ agar grout 


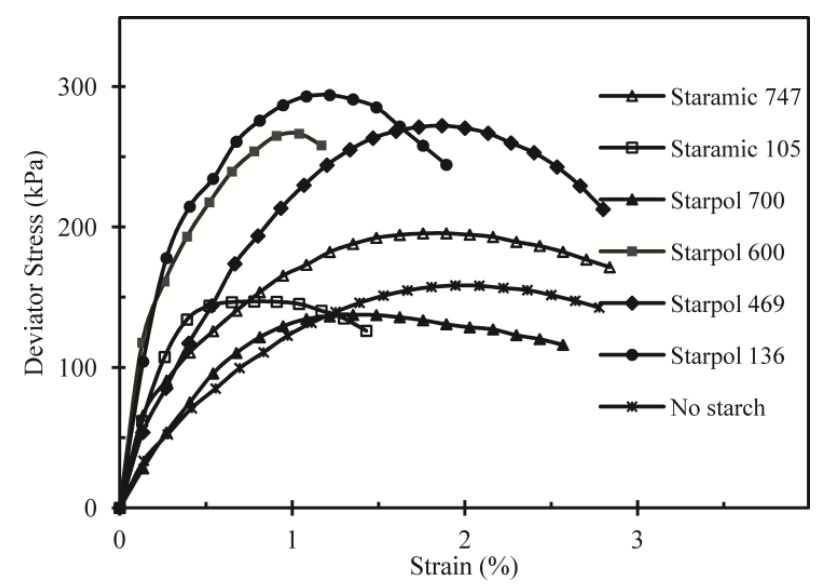

Fig 3. Unconfined compressive tests of sand treated with $1 \%$ agar and $0.5 \%$ starch

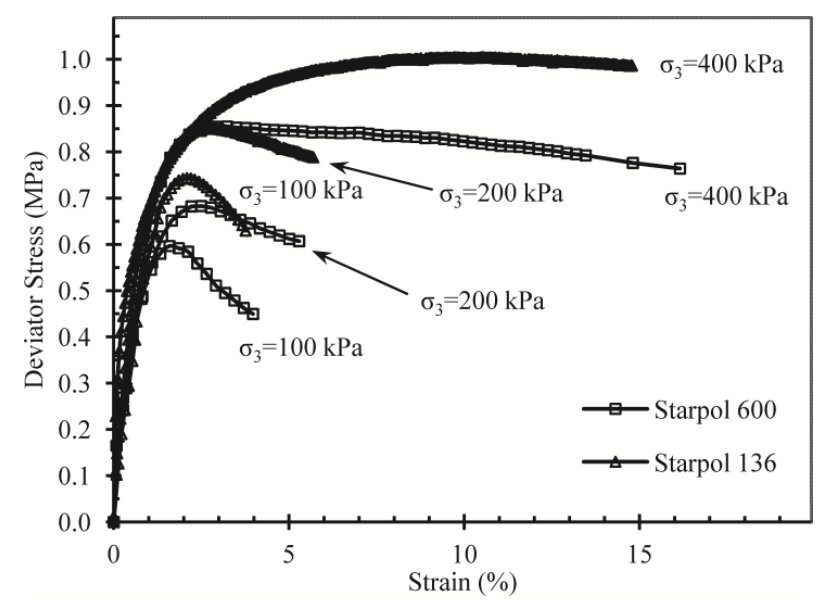

Fig 4. Triaxial compression tests of sand treated with $1 \%$ agar and $0.5 \%$ Starpol 600/1\% Starpol 136 

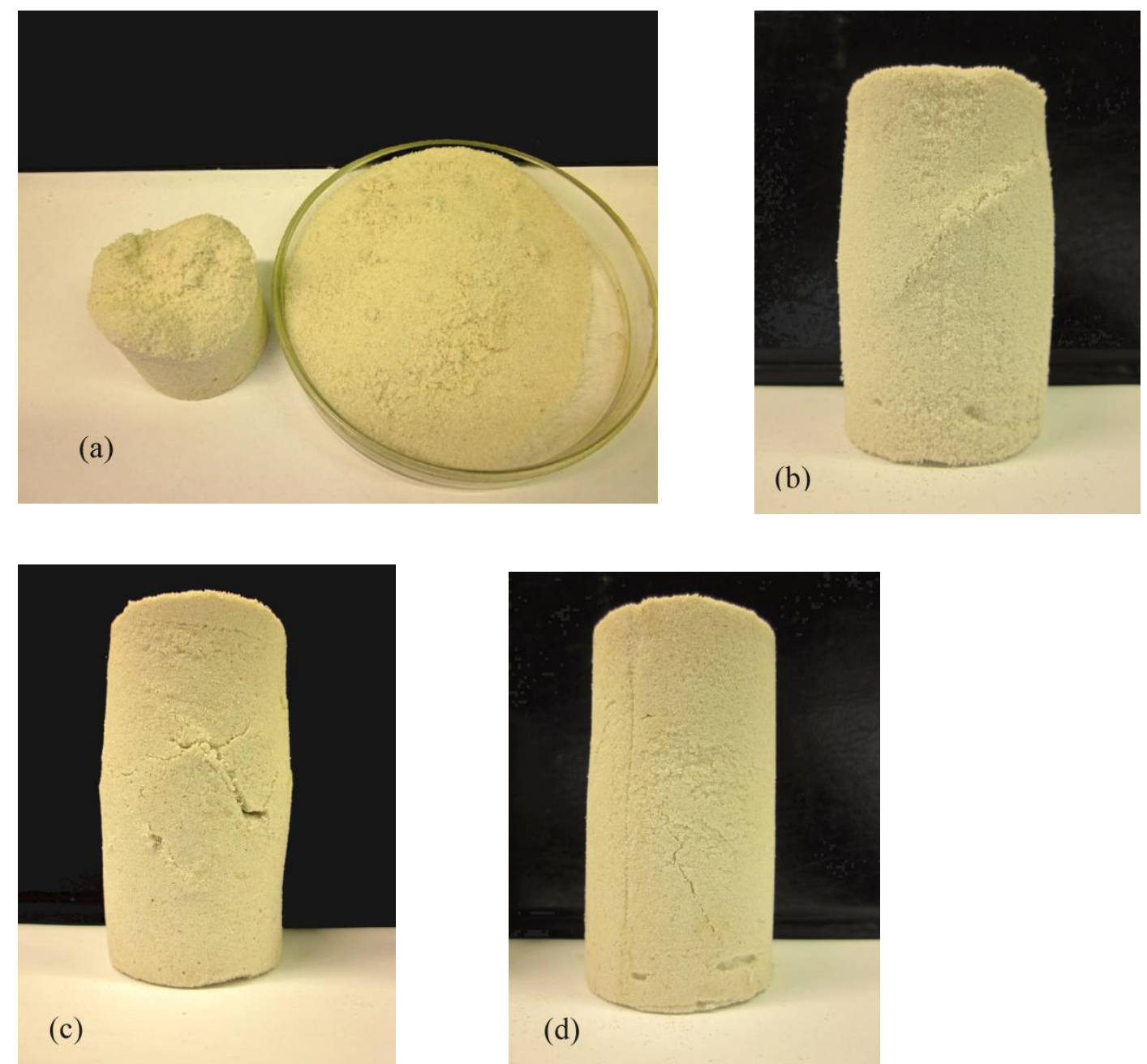

Fig 5. Shearing modes and strain at failure (peak deviator stress) in triaxial compression under a confining pressure of $100 \mathrm{kPa}$ : (a) $1.0 \%$ agar at $2 \%$ strain; (b) $2.0 \%$ agar at $2.5 \%$ strain; (c) $4.0 \%$ agar at 3\% strain; (d) $1.0 \%$ agar and $0.5 \%$ Starpol 600 at $1.6 \%$ strain 